\title{
AN EXPERIMENT TO MEASURE THE DIRECTION AND ENERGY SPECTRUM OF EXTRATERRESTRIAL GAMMA RAYS IN THE ENERGY RANGE 1-10 MeV FROM BALLOON ALTITUDES
}

\author{
A. J. DEAN \\ Laboratorio Fisica Cosmica e Tecnologie Relative del CNR Milano, Italy \\ and \\ A. BELLOMO, P. COFFARO, M. FATTA, G. GERARDI, F. MADONIA, \\ A. R USSO and L. SCARSI \\ Laboratorio Fisica Cosmica e Tecnologie Relative del CNR, Palermo, Italy'
}

\begin{abstract}
Problems of making a device for directional measurements of extraterrestrial gamma rays in the $\mathrm{MeV}$-range are discussed - especially background counting rates. A detector system is described which is planned to be flown in a series of balloon experiments in order to provide a better understanding of the sources of background and their elimination.
\end{abstract}

\section{Introduction}

Although experiments have been carried out over a period of more than a decade, $\gamma$-ray astronomy in the $\mathrm{MeV}$ range of energies has progressed very little. The only significant measurements made to date are derived from non-directional detecters in deep space probes (Metzger et al., 1964; Vette, 1970). These experiments have indicated a bump in the spectrum of the diffuse background in the region of a few MeV. Three major interpretations have been made on this effect (Clayton, 1969; Stecker and Silk, 1969; Anand, 1970).

To date measurements exist for several point sources at X-rays frequencies, where the intensity and spectral shape have been measured with varying degrees of accuracy over the limited bandwith of the devices used. At present there are several spark chamber experiments under construction designed to measure either the spectral shape, or if not, to give an integral point for the X-ray sources in the region of $50 \mathrm{MeV}$. Two discrete sources, besides the diffuse background radiation, have already been measured in both the X-ray region and at $50 \mathrm{MeV}$. These are the galactic plane (Cooke et al., 1969; Clark et al., 1968) and the pulsar in the Crab Nebula (Fritz et al., 1969; Fishman et al., 1969; Vasseur et al., 1970).

In order to clarify the generation mechanism in the case of the sources so far observed more measurements at different frequencies are required. This is not only true for the sources so far detected at X-ray wavelength alone, where differentiation is difficult at present between such mechanisms as thermal bremsstrahlung and power law spectra, but also in the cases where finite measurements have been made in the region of $50 \mathrm{MeV}$. The measurements of Clark et al. on the diffuse background has not enabled an isolation of the Clayton or Stecker hypotheses or indicated that some other mechanism must exist. A similar situation exists for the Crab pulsar. Here the 
spark chamber measurements have indicated that a change of slope exists in the pulsed spectrum at energies higher than the X-ray region of the spectrum. There are two theories which predict a change in slope (Apparao, 1970; Dean and Turner, 1971). These questions may be settled if directional measurements are made at $\mathrm{MeV}$ energies.

In this paper some of the problems of making such a device are discussed together with a series of balloon experiments which will enable the construction of a more advanced experiment.

At present the required technique is not well understood. Poor directionality may be obtained from the Compton effect. Collimation of the $\gamma$ rays in the $\mathrm{MeV}$ range is necessarily massive. In any case the collimation system must be made more sophisticated than to merely surround the detector with a stopping material, as attempts to do this have resulted in increased background counting rates (Jones, 1961; Allen et al.. 1968; Chapman et al., 1968). Thus to design a suitable shielding system in order to perform $\mathrm{MeV} \gamma$-ray astronomy a thorough understanding of the sources of background counting rates must be first obtained.

\section{Sources of Background Counting Rates}

The major sources of background counts for a collimation $\gamma$-ray detector, similar to that shown in Figure 1, are discussed below.

\subsection{Charged COSMIC RaYS}

Events arising from the passage of charged particles through the $\gamma$-ray sensitive region may be eliminated by the use of a high efficiency anticoincidence counter which completely surrounds the experiment.

\subsection{ATMOSPHERIC $\gamma$ RAYS}

A thickness, $x$, of shielding material may be used to reduce the background counting rate from low energy atmospheric $\gamma$ rays. The fraction of $\gamma$ rays which penetrates this shield is given by:

$$
I_{x} / I_{0}=B\left(E_{\gamma}, x, \text { material }\right) \cdot \exp \left\{-\mu\left(E_{\gamma}, \text { material }\right) x\right\},
$$

where $B$ is the build-up factor. The mass absorbtion coefficient, $\mu$, has a minimum value, for high $z$ elements, in the region of $3 \mathrm{MeV}$, making an effective collimation system for $\mathrm{MeV} \gamma$ rays necessarily massive. This situation is further aggravated when the effect of the build-up factor is integrated over the spectrum of the atmospheric $\gamma$ rays, Figure $2 \mathrm{a}$ as the $\gamma$ rays in the $\mathrm{MeV}$ range generated from higher energy $\gamma$ rays tend to be preferentially transmitted. To reduce this type of background counting rate the shield may be made active (i.e. sensitive over its whole volume to charged particles).

In order to have such an active shield of sufficient stopping power without using a prohibitive amount of expensive Cs I crystals, a compromise high $z$ stopping material scintillation plastic 'sandwich' arrangement may be used as shown schematically 
in Figure 1. The effect of this sandwich arrangement on the reduction of secondary $\gamma$ rays with energy $1<E_{\gamma}<10 \mathrm{MeV}$ is shown in Figure $2 \mathrm{~b}$.

\subsection{NEUTRON INDUCED BACKGROUND}

Neutrons interact in the material of the experiment package to give background counts with the following interactions: slow neutrons - capture $\gamma$ rays; fast neutrons knock-on protons, inelastic collisions giving energetic charged particles, inelastic interactions giving $\gamma$ rays, thermalization to give capture $\gamma$ rays. The sources of neutrons are the Earth's atmosphere and 'stars' generated in the material of the experiment. A high $z$ shield does little to attenuate neutron fluxes.

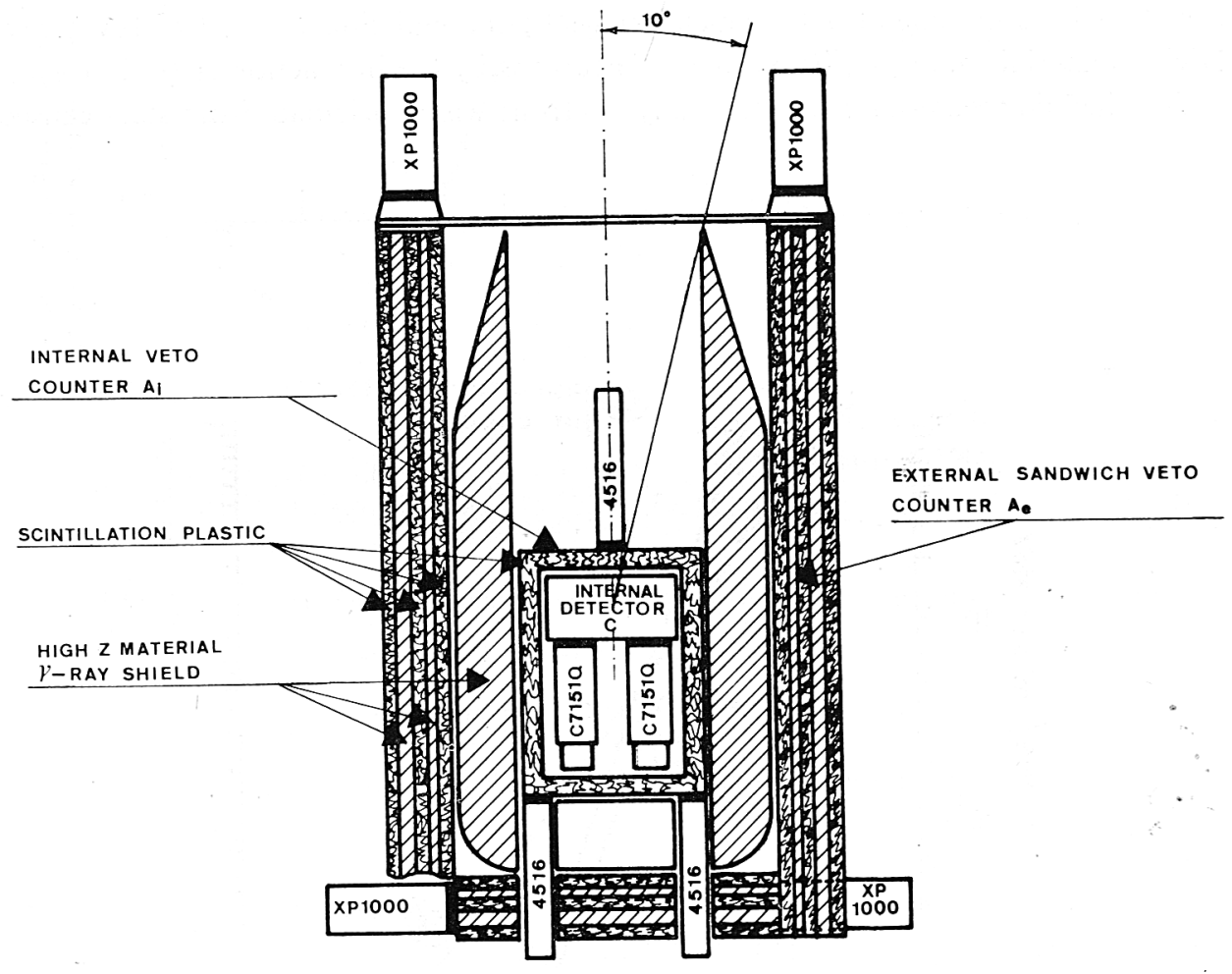

Fig. 1.

Atmospheric thermal neutrons may be easily eliminated by surrounding the experimental package with a boron loaded shield. Boron in various chemical compounds may be distributed throughout the material of the experiment in a sufficient concentration to effectively complete with the thermal neutron-decay captures of the nuclei of the various construction materials. The other mode of decay of the interaction is

$$
n+\mathrm{B}^{10} \rightarrow \mathrm{Li}^{7 *}+x \rightarrow \mathrm{Li}^{7} x+\gamma(0.48 \mathrm{MeV})
$$


which sets a lower threshold on the experiment range if this $\gamma$-ray line cannot be isolated. Lead and bismuth have the lowest thermal neutron capture cross-sections of the high density materials available. $\left\{\sigma_{T}(n, \gamma)_{p b}=22 \mathrm{mb} \times \sigma_{T}(n, \gamma)_{B_{i}}=17 \mathrm{mb}\right\}$.

If no moderator is used to attenuate the fast atmospheric neutron fluxes, the system must be designed to be as insensitive to fast neutrons as possible, by a choice of materials which minimize gamma-production by fast neutrons, and the use of a detector that differentiates between electrons and protons of about the same energy.

Of the higher $z$ elements, bismuth is very insensitive to the production of $\gamma$ rays by fast neutrons. Bismuth exists mainly in one isotope and the energy level scheme of the exited states is simple.

Lead generates several $\gamma$ ray lines in the $\mathrm{MeV}$ range and has a metastable state, $\mathrm{Pb}^{207 *}$, with a lifetime of $0.8 \mathrm{~s}$ which decays by the emission of a $1.07 \mathrm{MeV} \gamma$ ray. High $z$ materials multiply the number of neutrons by the interaction $\operatorname{Ai}(n, 2 n) A_{i-1}$.

The shielding material generates many neutrons which originate from stars caused

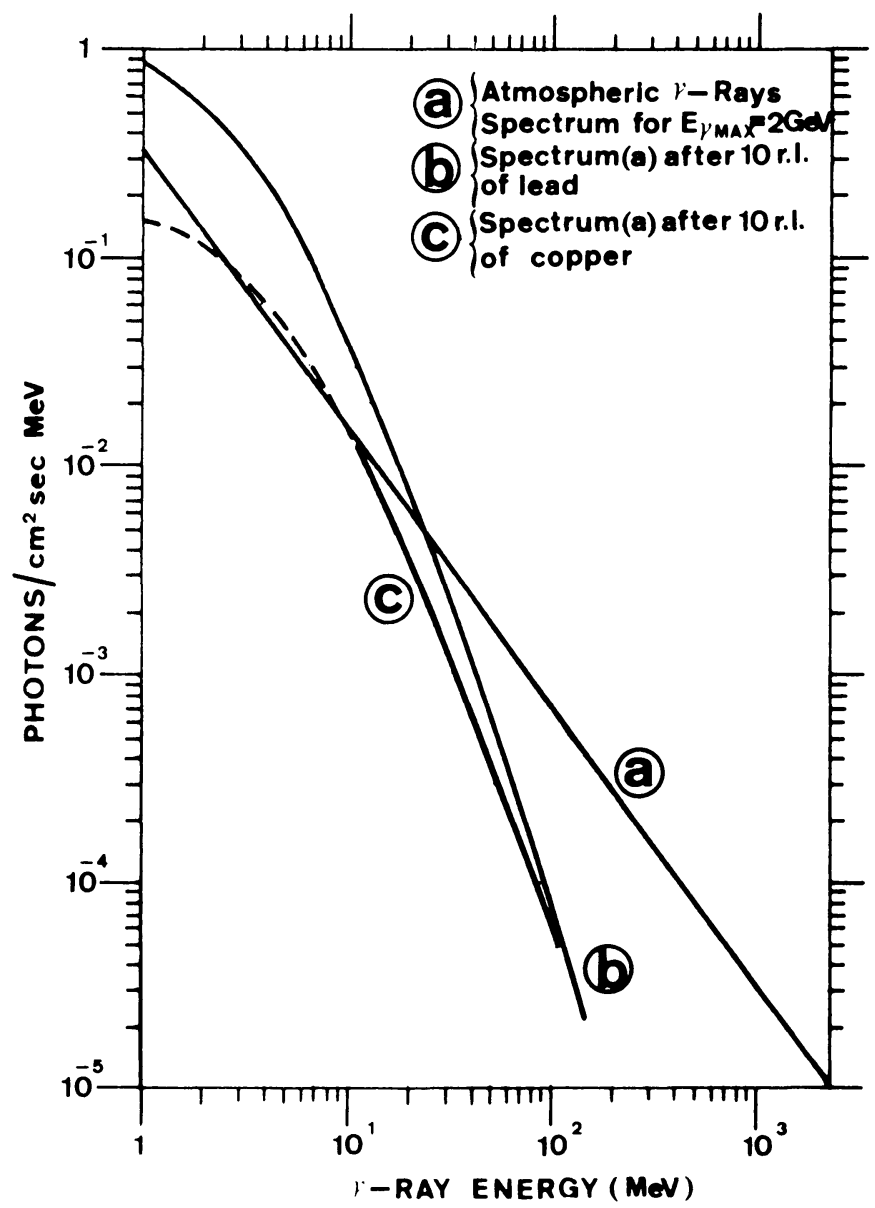

Fig. 2a. Transition curves for atmospheric ; rays in lead and copper. 


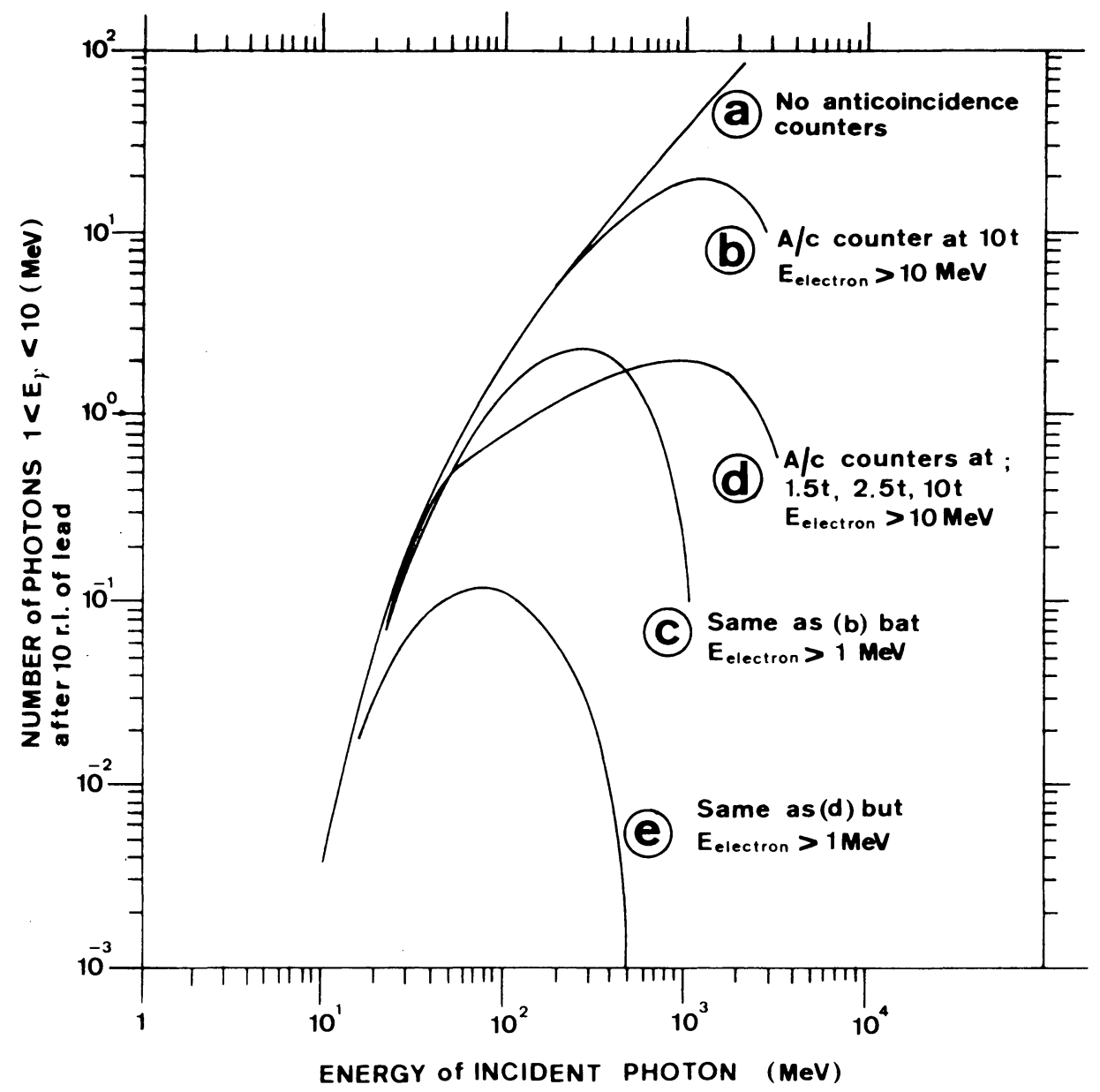

Fig. 2b. Effect of Sandwich anticoincidence shielding system.

by interactions of the cosmic ray flux. Higher $z$ materials produce more neutrons per star ( $\mathrm{Pb}$ gives $\sim 8 n / \mathrm{star})$. The number of neutrons per incident cosmic ray is a function of the thickness of the material because the pions produced in the interactions may themselves generate more nuclear interactions.

The background events due to star neutrons which give prompt counts in the central detecter are easily eliminated by the veto pulse derived from the initial cosmic ray or one of the product pions. Events from slow star neutrons, fast ones thermalized in the material of the experiment and inelastic interactions giving delayed gamma-rays cannot be covered by a veto pulse which must have a finite length in order to prevent complete blockage of the gamma-ray event counting channel.

In order to reduce this source of background the materials used throughout the experimental payload must be chosen with care. 
The problem of the background counting rates due to neutrons generated in the material of the detector arrangement is common to both satellite and balloon experiments. For a satellite payload the effects of the Earth's atmosphere may be minimized by a suitable choice of orbit. The problem of local neutron and $\gamma$-ray production may be reduced by a control over the total payload geometry; otherwise the incorporation of such an experiment in a massive satellite can have the same effect as to fly the device in the Earth's atmosphere, thus losing one of the advantages of a satellite platform.

A series of balloon experiments have been planned to provide a better understanding of the sources of background and their elimination.

\section{Description of the Experimental Arrangement}

\subsection{Central Detector}

A schematic diagram of the experimental arrangement is shown in Figure 1. C is the central $\gamma$-ray device and is a cylinder of radius $5.7 \mathrm{~cm}$ with a height of $5 \mathrm{~cm}$. The detecter is viewed by four RCA type C7151Q photomultipliers, chosen for their short overall length, high quantum efficiency, and fast response. Following the principle of boron loading, as discussed above, three basic types of detector may be used.

(i) A Čerenkov device which is a highly transparent compound containing boron. The Cerenkov velocity threshold will automatically eliminate knock-on protons and the $2.81 \mathrm{MeV} \propto$ particles. Several suitable boron compounds exist in nature but are rare and therefore expensive. Two compounds are at present under consideration, dehydrolized Boric acid $\left(\mathrm{HBO}_{2}\right)$ and Trimethyl Borate $\left(\mathrm{B}\left(\mathrm{OCH}_{3}\right)_{3}\right)$.

Preliminary tests have been made with a 'perspex' detector, painted white to give a diffusive light collection system. With the threshold set at the one photoelectron level on each photomultiplier the efficient detection of $\operatorname{Co}^{60} \gamma$ rays $\left(E_{\gamma}=1.1\right.$ and 1.3 $\mathrm{MeV}$ ) was achieved. Their pulse height distribution is shown in Figure 3. The disadvantages of a Cerenkov system are the low photoelectron number and the poor energy resolution inherent in such a system.

(ii) A Boron loaded scintillation counter in which the Compton and pair production electrons may be distinguished from knock-on protons and $\alpha$ particles by a combination of pulse height and pulse shape analysis. Such a system, since it is ionization sensitive, will give a better energy resolution for the $\gamma$ rays. Boron loaded liquid scintillators are commercially available, NE-311 and NE-321. At present the separation efficiency and detection proporties of both the liquids are under study in the laboratory.

(iii) A scintillation counter in which the scintillation material is isolated from a transparent boron compound by enough material to stop the $2.81 \mathrm{MeV} \propto$ particles. Such a device should have a uniform response to $\gamma$ rays and be insensitive to thermal neutrons. A system is at present under construction in which each sandwich element consists of the following: $1 \mathrm{~mm} \mathrm{NE}-102 \mathrm{~A}$ scintillation plastic, $200 \mu \mathrm{HBO}_{2}, 12 \mu$ mylar. The dimensions were chosen according to the additional requirements that 
there must be enough $\mathrm{B}^{10}$ atoms to compete effectively with the $\gamma$ capture processes in the remaining material and that the total thickness of the one sandwich element must be about the same as the range of the Compton electrons $(<500 \mathrm{keV})$ at the lower end of the experiment bandwith. In this way the pulse height distribution is not unnecessarily broadened.

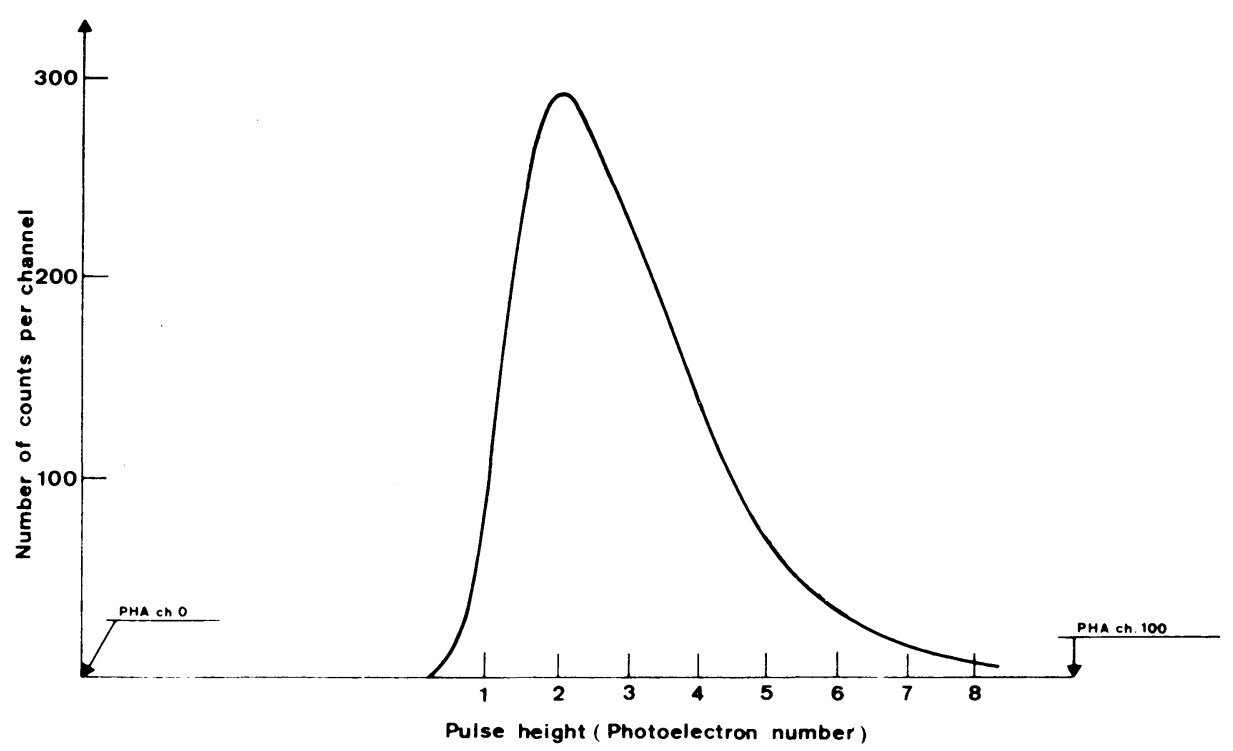

Fig. 3. Pulse height distribution of Co $\gamma$ rays in perspex.

\subsection{THE ANTICOINCIDENCE COUNTERS AND SHIELDING SYSTEM}

The inner anticoincidence counter is a closed hollow cylinder of plastic scintillator, with a wall thickness of $1 \mathrm{~cm}$. It is viewed by four RCA 4516 photomultipliers, two at each of the cylinder and displaced by $90 \mathrm{deg}$ around the circumference.

The 'sandwich' anticoincidence arrangement is made from a series of concentric cylindrical scintillators interspaced with high $z$ elements. The scintillators are viewed by six photomultipliers spaced around the circumference, each photomultiplier views all the 'Sandwich' elements. The bottom element is viewed by a further two photomultipliers. The light collection is by total internal reflection. The total experiment payload is surrounded by a boron compound in order to provide a shield against atmospheric thermal neutrons.

A cylindrical aperture through the shielding material provides the collimation and has a half angle of about $10 \mathrm{deg}$. This angle was chosen to be compatible with the following factors: (a) It must be greater than the steering errors to avoid loss of observation time due to tracking problems. (b) It must provide a reasonable angular resolution of sources. (c) It must be less than the angle at which the number of $\gamma$ rays 


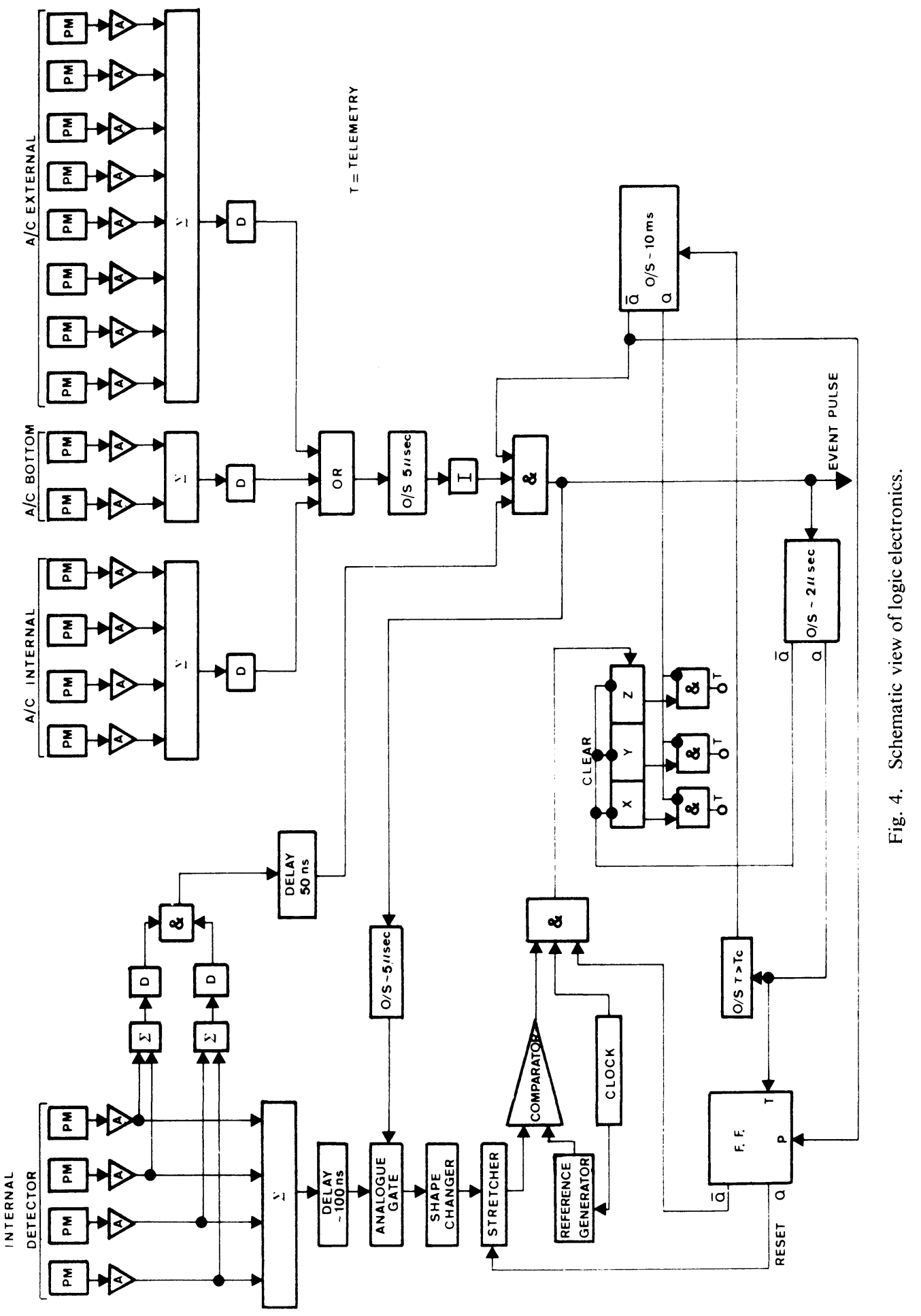


that enter through the aperture are equal to the background rate from all the other sources, otherwise the statistical accuracy will be reduced.

\section{The Electronic System}

A schematic block diagram of the electronic system is shown in Figure 4. A $\gamma$-ray event is defined by the following requirement:

$$
\left(C_{1}+C_{2}\right) \cdot\left(C_{3}+C_{4}\right) \cdot \bar{A}_{\mathrm{int}} \cdot \bar{A}_{\mathrm{ext}} .
$$

All photomultipliers and electronic circuits used in the event selection logic are compatible with the use of $10 \mathrm{~ns}$ pulses. The same operational amplifier is used in the amplification stages as well as for the algebraic summations. Tunnel diode discriminator circuits are employed. The remaining logic is made from integrated circuits.

The length of the anticoincidence pulse is made as long as possible consistent with the noise pulse rates and particle counting rates at altitude. The dead time of the experiment after each event depends on the telemetry channels used.

Upon the selection of a $\gamma$-ray event, a pulse height analysis is performed on the algebraic sum $\left(C_{1}+C_{2}+C_{3}+C_{4}\right)$. This is done by first converting the $10 \mathrm{~ns}$ long summed pulse into an electrical signal with a rise time of $\sim 300 \mathrm{~ns}$ and a fall time of $\sim 3 \mu \mathrm{s}$. This pulse is then analysed in a stretcher/comparator circuit so that the energy channels have a width which has a logarithmic dependence on the $\gamma$-ray energy. The narrower channels are used for the low energy $\gamma$ rays where more events are expected per $\mathrm{MeV}$. The number of channels available may be changed simply by successively doubling the clock frequency and adding an extra telemetry channel, if parallel readout is used. The number of energy channels available is $\left(2^{n}-1\right)$ where $n$ is the number of telemetered bits.

\section{Flight Programme}

It is difficult to estimate with a high degree of accuracy the relative importance of the various background counting rates discussed above. To understand better this problem a series of flights is planned in which different arrangements of the shielding system and type of central detector will be studied. Different $\gamma$-ray attenuation systems will be studied ranging from the very high $z$ materials, such as lead and bismuth, to intermediate materials (iron, copper etc.) or combinations of a high and low $z$ material. Boron loaded central detectors will be flown and also their non boron-loaded counterparts. Thus for such systems the intensity and spectral shape of the background counting rate may be compared with the calculated values. With this knowledge a system optimized to give the lowest background rate may be designed, with either balloon or satellite payloads in mind. The satellite case is somewhat simpler if a vehicle is chosen in which a large fraction of the satellite is the experiment, so that good control over the materials used and their geometrical arrangement may be achieved. 


\section{References}

Allen Womack, E. and Overbeck, J. W.: 1968, Bul. Am. Phys. Soc. 13, 1396.

Anand, K. C.: 1970 (preprint).

Apparao, K. M. V. and Hoffman, J.: 1970, Astrophys. Letters 5, 25.

Chapman, G. T., Macklin, R. L., Gibbons, J. H., and Nutt, R.: 1968, Bull. Am. Phys. Soc. 13, 695.

Clark, G. W., Garmire, G. P., and Kraushaar, W. L.: 1968, Astrophys J. 153, L203.

Clayton, D. D.: 1969, 11 th I.C.C.R., Budapest.

Cooke, B. A., Griffiths, R. E., and Pounds, K. A.: 1969, Nature 224, 134.

Dean, A. J. and Turner, M. J. L.: 1971, (to be published).

Fishman, G. J., Harnden, F. R., and Haymess, R. C. 1969, Astrophy's. J. 156, L107.

Fritz, G., Henry, R. C., Meekins, F. J., Chubb, T. A., and Friedman, H.: 1969, Science 164, 709.

Jones, F. C.: 1961, J. Geophys. Res. 66, 2029.

Metzger, A. E., Anderson, E. C., Van Dilla, M. A., and Arnold, J. R.: 1964, Nature 204, 766.

Stecker, F. W. and Silk, J.: 1969, Nature 221, 1229.

Vasseur, J., Paul, J., Parlier, B., Leray, J. P., Forichon, M., Agrinier, B., Boella, G., Maraschi, L., Treves, A., Buccheri, R., and Scarsi, L.: 1970, Nature 226, 534.

Vette, J. I., Gruber, D., Matteson, J. L., and Peterson, L. E.: 1970, in L. Gratton (ed.), 'Non-Solar $\mathrm{X}$ - and Gamma-Ray Astronomy', I AU Symp. 37, 335. 